\title{
A Rare Polypoid Lesion of The Palatine Tonsil :Lymphangiomatous Polyp
}

\author{
Sunitha Ramachandra*, Lakshmi Rao and Masoud Al Kindi
}

Department of Pathology, Armed Forces Hospital, Muscat, Sultanate of Oman

\section{ABSTRACT}

We report an unusual benign lesion of the tonsil noticed as a mass in the oral cavity, in a 17-year-old male. Excision of the lesion showed proliferation of endothelial-lined lymph channels with luminal lymphocytes surrounded by loose fibrous tissue consistent with tonsillar lymphangiomatous polyp (LP). Though a rare lesion with only a very few reported cases, arriving at a correct diagnosis is not difficult.

\section{Keywords: Tonsillar Polyp, Lymohangiomatous Lesion of Oral Cavity, Hamartoma of The Tonsil.}

\section{Introduction}

Lymphangiomatous lesions are commonly seen in the head and neck. ${ }^{1}$ They arise in the skin and subcutaneous tissue. ${ }^{1}$ Lymphangiomatous polyps can be seen in the larynx, parotid gland, mouth, tongue and gastrointestinal tract. ${ }^{1,2}$ Tonsils are a less common site for lymphangiomatous lesions. ${ }^{3}$ LPs are considered as hamartomatous lesions as they show proliferation of elements derived from the local tissue. $^{4}$

\section{Case History}

A 17-year-old male presented with history of recurrent tonsillitis and noticed a mass in the oral cavity for the last two years. No complaints of obstructive symptoms or change in the size or texture of the mass. Clinical examination showed a pedunculated lesion arising from the superior pole of the right tonsil with similar morphological appearance as with tonsil and a grade 2 tonsillitis (bilateral). The case was managed by tonsillectomy along with excision of the pedunculated lesion. The specimen was sent for histopathological examination. Grossly, the specimen showed a polypoid lesion measuring $4 \times 2 \times 1.5 \mathrm{~cm}$ attached to the tonsil. Slicing showed a solid, pale brown surface (Fig 1). Microscopically, the polyp was covered by stratified squamous epithelium with mild acanthosis overlying the loose fibrocollagenous stroma with dilated lymphatics, a few vascular channels and dilated, congested blood vessels surrounded by occasional plasma cells, lymphocytes and lymphoid aggregates along with focal areas of stromal fibrosis and lipoid infiltration (Fig 2). Sections from the tonsils showed crypt inflammation containing microbial colonies and lymphoid hyperplasia consistent with chronic tonsillitis. Immunohistochemistry (IHC) showed lymphatic channels positive for Factor VIIIrelated antigen and CD 31 with negative CD 34 (Fig 3).
A diagnosis of tonsillar LP with chronic tonsillitis of the adjacent and contralateral tonsils was rendered.

\section{Discussion}

A lymphangiomatous polyp (LP) is a rare benign lesion arising from the tonsil. Lymphangiomas or lymphatic malformation are commonly seen in the head and neck region and the gastro-intestinal tract. ${ }^{2}$ Other sites of predilection include mouth, tongue, larynx and parotid gland. Palatine tonsil is an uncommon site. Its actual incidence is unknown because of its confusing histologic nomenclature reported with various alternative terminologies such as angioma, angiofibroma, polypoid lymphangioma of the tonsil, hamartomatous tonsillar polyp and lymphoid polyp. ${ }^{1}$ Also, there is a lack of awareness of this rare benign condition among clinicians. ${ }^{2}$ To date, around 30 cases of LP of the palatine tonsil have been reported. ${ }^{1}$ It is seen in children and young adults. ${ }^{1}$ The single largest case study has shown on average a mean age of 25 years..$^{5}$ It is usually unilateral with no size or gender predilection., ${ }^{2,5}$ The symptoms are dependent on the size of the polyps. Common presenting complaints include sore throat, dysphagia, dyspnoea, a sense of fullness or a foreign body sensation. ${ }^{1,5}$ Snoring, cough, aspiration and eructations can occur depending on the size. ${ }^{4}$

Clinical examination is important for the diagnosis and shows the polyp attached to the tonsil, tonsillar bed or to the wall of the hypopharynx, pedunculated and lobulated with a smooth surface. ${ }^{4}$ Aetiology remains unclear, although chronic inflammation and obstruction of lymphatic channels was the possible suggested mechanism in relation to chronic tonsillitis. Our case had history of recurrent tonsillitis. LPs are considered to be hamartomatous lesions as they show haphazard proliferation of tonsil lymphoid and 


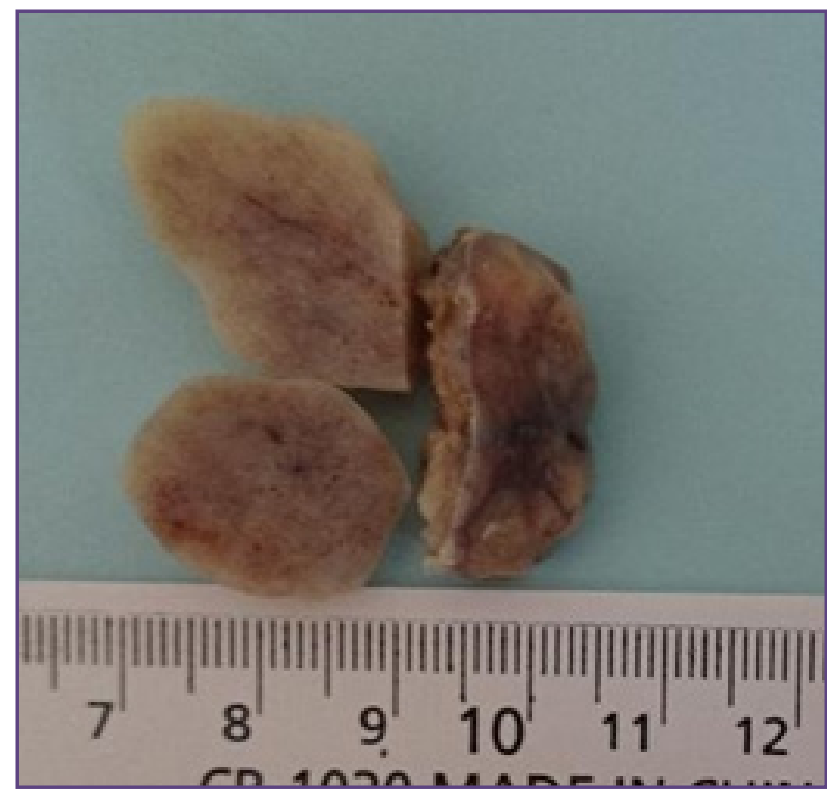

Fig. 1 :Gross picture of bisected polyp with attached tonsil.

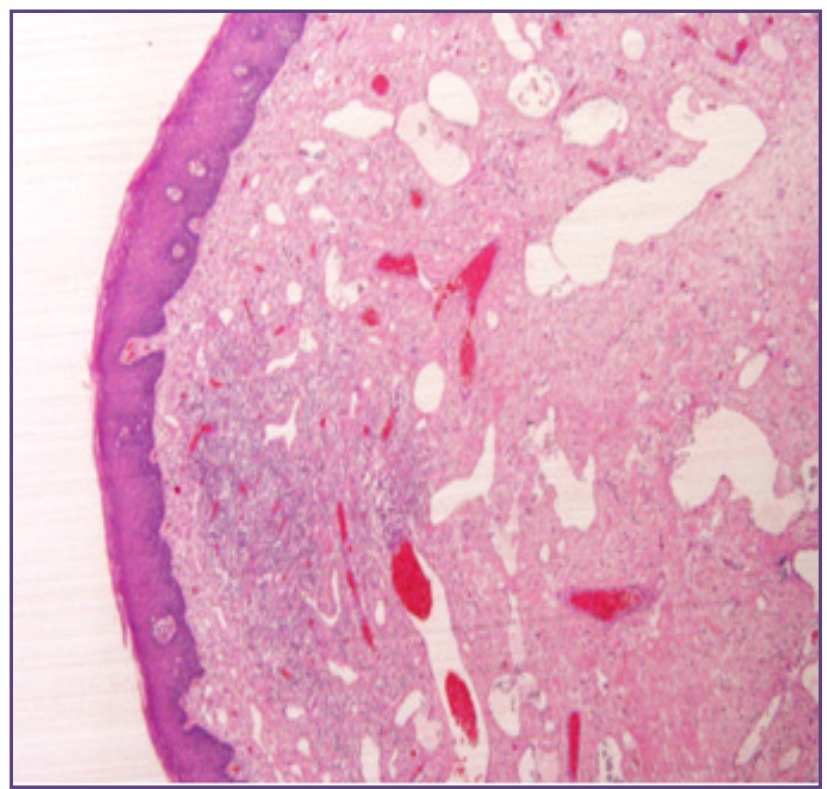

Fig. 2 : Polyp surface covered by stratified squamous epithelium overlying the stroma with lymphatic channels, blood vessels and lymphoid follicles. (Hematoxylin \& Eosin stain $x$ 4X).

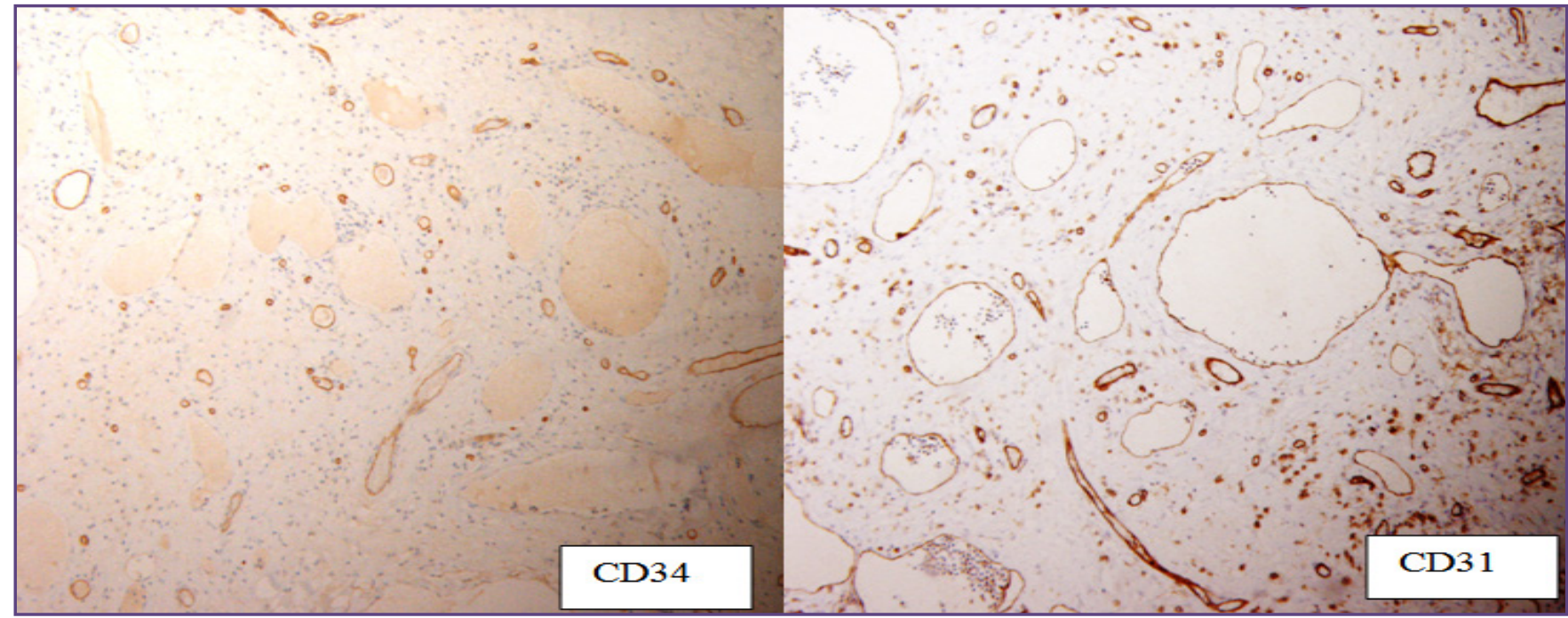

Fig. 3 : Lymphatic channels negative for CD 34 and positive for CD 31 along with vascular channels (IHC stain).

epithelial components that are native to the tonsil. ${ }^{4}$ Grossly, LPs show a similar morphological appearance as tonsils ranging in size from 0.5 to $3.8 \mathrm{~cm}$ with white, tan or yellow cut surface. ${ }^{5}$ Histologically, they are covered by either stratified squamous epithelium or respiratory epithelium and may show epithelial hyperplasia. ${ }^{3}$ Proliferation of lymphovascular channels with luminal lymphocytes and proteinaceous fluid is the main diagnostic feature. Stroma showed collagen, adipose tissue and lymphocytic infiltration. ${ }^{3}$ This polypoid benign condition needs to be differentiated from juvenile angiofibroma, fibroepithelial polyp, papilloma, arteriovenous malformation and lymphangioma.,

Clinically, juvenile angiofibromas occur in the nasopharynx with epistaxis, extensive growth and occasional bone erosion. Hence treated more aggressively to prevent recurrence, which shows staghorn-like vascular channels and a cellular stroma with stellate cells. ${ }^{5}$ Papillomas are usually exophytic, lacking lymphovascular proliferation. Lymphangiomas show widely ectatic lymph channels 
with luminal lymphocytes and proteinaceous fluid. Surgical excision is curative and can be combined with tonsillectomy in case of associated tonsillitis.

All lymphatic channels are enveloped by a thin wall of smooth muscle. They were uniformly reactive for Factor VIIIrelated antigen. ${ }^{3}$ CD 31 and CD 34 show variable expression. Our case was CD 34 negative and positive for Factor VIIIrelated Ag and CD 31. D240, Prox1 (prospero-related homeobox gene1) and VEGFR3 (vascular endothelial growth factor receptor) are lymphatic endothelial markers. ${ }^{6}$ Endothelial cells of venous malformations are negative for Prox $1^{6}$ but are positive for CD 31, CD 34 and VEGFR3. ${ }^{6}$ Nevertheless, IHC is not needed for definitive diagnosis. ${ }^{3}$

\section{Conclusion}

LP is a rare, benign condition of the palatine tonsil, considered as a hamartomatous lesion with proliferation of mature tissue components relative to the tonsil. Lack of awareness among clinicians and confusing histomorphology are a hindrance to measure its actual incidence. Simple excision offers complete care and IHC is not needed for its diagnosis.

\section{References}

1. Chen CS, Cheng CL. A pedunculated lymphangiomatous polyp of the palatine tonsil. A case report. Braz.j.otorhinolaryngol.2013 May/June;79(3):166-68

2. Park E., Pransky S. M., Malicki D. M., Hong P. Unilateral lymphangiomatous polyp of the palatine tonsil in a very young child: a clinicopathologic case report. Case Reports in Pediatrics. 2011;2011:3.

3. Ryu HS, Jung SY, Koh JS and Lee SS. Tonsillar Lymphangiomatous Polyp - Report of Two Cases. The Korean Journal of Pathology. 2006; 40: 381-4

4. Z A AL-Qudehy, Y AL-Nufaily, H Yagi. Tonsillar Lymphangiomatous Polyp, A Case Report and Literature Review. The Internet Journal of Otorhinolaryngology. 2013; 15(1).

5. Kardon DE, Wenig BM, Heffner DK and Thompson LDR. Tonsillar Lymphangiomatous Polyps: A Clinicopathologic Series of 26 Cases. Modern pathology.2000 Oct;13(10):1128-33.

6. Castro EC1, Galambos C. Prox-1 and VEGFR3 antibodies are superior to D2-40 in identifying endothelial cells of lymphatic malformations--a proposal of a new immunohistochemical panel to differentiate lymphatic from other vascular malformations. Pediatr Dev Pathol. 2009;12(3):187-94.

*Corresponding author:

Dr. Sunitha Ramachandra, Department of pathology, Armed forces Hospital, Al- khoud, PO Box 325, PC Seeb 111, Muscat, Oman.

Email: sunithamanjunath98@yahoo.co.in

Financial or other Competing Interests: None. 